\title{
JaponicusDB: Rapid deployment of a model organism database for an emerging model species
}

\author{
Kim M. Rutherford, ${ }^{1}$ Midori A. Harris, ${ }^{1}$ Snezhana Oliferenko ${ }^{2,3, *}$ and Valerie Wood ${ }^{1, *}$ \\ ${ }^{1}$ Department of Biochemistry, University of Cambridge, Cambridge CB2 1GA, UK, ${ }^{2}$ The Francis Crick Institute, London NW1 1 AT, UK \\ and ${ }^{3}$ Randall Centre for Cell and Molecular Biophysics, School of Basic and Medical Biosciences, King's College London, London SE1 1UL, \\ UK \\ *Corresponding author. snezhana.oliferenko@kcl.ac.uk (S.O.), vw253@cam.ac.uk (V.W.) \\ FOR PUBLISHER ONLY Received on Date Month Year; revised on Date Month Year; accepted on Date Month Year
}

\begin{abstract}
The fission yeast Schizosaccharomyces japonicus has recently emerged as a powerful system for studying the evolution of essential cellular processes, drawing on similarities as well as key differences between S. japonicus and the related, well-established model Schizosaccharomyces pombe. We have deployed the open-source, modular code and tools originally developed for PomBase, the $S$. pombe model organism database (MOD), to create JaponicusDB (www.japonicusdb.org), a new MOD dedicated to $S$. japonicus. By providing a central resource with ready access to a growing body of experimental data, ontology-based curation, seamless browsing and querying, and the ability to integrate new data with existing knowledge, JaponicusDB supports fission yeast biologists to a far greater extent than any other source of S. japonicus data. JaponicusDB thus enables $S$. japonicus researchers to realise the full potential of studying a newly emerging model species, and illustrates the widely applicable power and utility of harnessing reusable PomBase code to build a comprehensive, community-maintainable repository of species-relevant knowledge.
\end{abstract}

Key words: model organism database, Schizosaccharomyces japonicus, genome, annotation, curation, evolution

\begin{abstract}
Introduction
In recent years the fission yeast Schizosaccharomyces japonicus has emerged as a powerful system for studying evolutionary ${ }^{22}$ cell biology, via comparison to the related, well-established ${ }^{23}$ model eukaryote Schizosaccharomyces pombe. Although these ${ }^{24}$ two fission yeasts carry out conserved cell-level processes using ${ }^{25}$ similar gene complements, substantial physiological differences ${ }^{26}$ in the mechanisms underpinning cell growth and division (Aoki ${ }^{27}$ et al., 2011; Yam et al., 2011; Gu et al., 2015; Makarova et al., ${ }^{28}$ 2016), physiology (Okamoto et al., 2013; Kinnaer et al., 2019), ${ }^{29}$ and metabolism (Bulder, 1963; Kaino et al., 2018; Makarova ${ }^{30}$ et al., 2020) provide invaluable opportunities to study how ${ }^{31}$ processes accomplished by universally conserved gene products ${ }^{32}$ may diverge. Indeed, $S$. pombe and $S$. japonicus may be ${ }^{33}$ regarded together as a composite model system in which ${ }^{34}$ conserved processes, gene products, and associated phenotypes ${ }^{35}$ can be compared side by side (Gu and Oliferenko, 2015; Russell ${ }^{36}$ et al., 2017; Oliferenko, 2018). Furthermore, S. japonicus can ${ }^{37}$ be used as a standalone model organism for studying biological ${ }^{38}$
\end{abstract}

processes not apparent or readily tractable in other model yeasts, such as nuclear envelope breakdown and reassembly (Yam et al., 2013; Pieper et al., 2020), cellular geometry scaling ( $\mathrm{Gu}$ and Oliferenko, 2019) and quorum sensing (Gómez-Gil et al., 2019).

The current trajectory of $S$. japonicus research also aptly illustrates how the demands placed on species-specific data resources grow and change as an organism becomes established as a model. To date, the $S$. japonicus genome sequence and associated computationally generated annotation available from existing species-neutral data aggregators and repositories, such as the INSDC databases (https://www.insdc.org/), Ensembl Genomes (Howe et al., 2020), UniProtKB/TrEMBL (The UniProt Consortium, 2020), and FungiDB (Basenko et al., 2018), has sufficed for the early stages of research in this model organism. As a model system matures, however, researchers move beyond simple exploratory screens to designing more ambitious research programs that generate heterogeneous genespecific molecular data for entire processes - a stage that $S$. 
japonicus research is now reaching. To accommodate growing 100 bodies of literature and data, and to realise the full potential of coordinated studies investigating the divergent biology of fission yeasts, the accumulated results of $S$. japonicus experiments must be made readily available to the research community in ${ }_{104}$ the expertly curated and integrated state provided only by $\mathrm{a}_{105}$ model organism database (MOD; Oliver et al. 2016; Lipshitz 2021).

To address the urgent need for MOD infrastructure and ${ }^{107}$ services for S. japonicus, we have created JaponicusDB using ${ }_{109}$ the suite of open-source modular, customizable tools and code originally developed for PomBase, the $S$. pombe MOD (Lock et al. 2018 and Harris et al. in this issue). The PomBase database system was designed to facilitate re-use for emerging 111 model species and comprises an online curation environment (Canto; Rutherford et al. 2014), a curation database using $_{113}$ the Chado schema (Mungall et al., 2007), and code to import data and generate a website that features intuitive displays, $a_{115}$ versatile query system, a genome browser (JBrowse; Buels et al. 2016), and support for daily data updates. Here, we describe the initial configuration and population of JaponicusDB and ${ }^{116}$ report on its current status. We summarise a round of manual ${ }^{117}$ curation in which we corrected and updated gene structure ${ }^{118}$ predictions, named genes, improved ortholog detection, and ${ }^{119}$ provided a greatly improved corpus of Gene Ontology (GO; The ${ }^{120}$ Gene Ontology Consortium 2000, 2021) annotation. Because ${ }^{121}$ community curation using Canto has proven successful in ${ }^{122}$ enhancing $S$. pombe literature curation (Lock et al., 2020), we ${ }^{123}$ have launched an analogous community curation approach for S. japonicus as a core part of JaponicusDB.

Now that both PomBase and JaponicusDB use the same $_{125}$ intuitive database system and curation environment, all fission 126 yeast researchers can easily carry out numerous activities that $t_{127}$ would not otherwise be feasible. Researchers can curate detailed 128 data from small-scale experiments, including phenotypes, modifications, molecular functions, interactions, and processes; display data from publication-based curation rapidly and ${ }_{130}^{129}$ conveniently; query curated data via simple, intuitive tools; and ${ }^{130}$ compare lists of genes identified in specific experiments with comprehensively curated lists in an intuitive and meaningful ${ }_{133}^{132}$ way. The rapid deployment of JaponicusDB showcases how ${ }^{133}$ reusing the PomBase system brings immediate benefits to $\mathrm{a}_{135}^{134}$ model organism community in exchange for very reasonable ${ }_{136}^{135}$ input funds and effort.

\section{Methods}

JaponicusDB shares a codebase with PomBase, which is ${ }^{141}$ used to build the database, load the data and run the website. All database-specific differences are captured in143 configuration files. The entire codebase is available from the144 PomBase GitHub organisation (https://github.com/pombase/), 145 and the JaponicusDB configuration files and manual curation146 in a separate GitHub organisation (https://github.com/147 japonicusdb/). Now that the initial setup is complete,148 JaponicusDB can be maintained almost entirely by editing files149 stored in GitHub repositories; anyone with a GitHub account150 can thus be authorised to make corrections and other changes.151 The database and website are automatically updated daily152 using the latest code, ontology versions, configuration and data153 files. Problems detected during this process are recorded to154 publicly visible $\log$ files to allow remote users to investigate155 issues.

\section{Database initialisation}

The first step in each daily update is to initialise a PostgreSQL database with an empty Chado schema (Mungall et al., 2007) and populate it with the required ontologies [Gene Ontology (GO; The Gene Ontology Consortium 2000, 2021), the Fission Yeast Phenotype Ontology (FYPO; Harris et al. 2013), the Sequence Ontology (SO; Eilbeck et al. 2005), the chemical ontology ChEBI (Hastings et al., 2016), the protein modification ontology PSI-MOD (Montecchi-Palazzi et al., 2008), and the Relations Ontology (RO; https://oborel. github.io/)].

\section{Preparing datasets}

We prepared data files for the first JaponicusDB load as described below. As noted above, with the exception of external files from InterPro and GOA, all annotation files are now maintained in GitHub repositories.

\section{Genome}

The S. japonicus genome contigs (EMBL:KE651166-KE651197; Rhind et al. 2011) and mitochondrial genome (EMBL:AF547983; Bullerwell et al. 2003) were downloaded from the European Nucleotide Archive (ENA; Harrison et al. 2021). Genome locus tags were repurposed to provide systematic identifiers, and identifiers (SJAGMIT_01-SJAGMIT_37) were minted for the mitochondrial genome.

\section{Transposons and LTRs}

All transposons, transposon-related fragments and LTRs reported by Rhind et al. (2011) were added to the sequence contigs, assigned IDs in the range SJATN_00001-SJATN_00265, and loaded into the $S$. japonicus JBrowse instance.

\section{Orthologs}

UniProtKB (The UniProt Consortium, 2020) records were downloaded using a query for taxon ID 402676 (the sequenced strain). Gene names applied by UniProtKB or via ENA submissions were imported. These were augmented by automated transfer of $S$. pombe gene names and products from PomBase where there is a one-to-one ortholog. Automatically transferred names are updated daily, with a configurable provision to override any unsuitable gene names or product descriptions. This configuration file also supplies manually curated names and product descriptions for paralogs, which we resolved based on synteny, and for genes with complicated orthology relationships, for which we used protein family membership to guide our decisions.

S. pombe orthologs. The S. japonicus-S. pombe orthologs identified by Rhind et al. (2011) for 4302 gene products were imported. These predictions were supplemented with orthologs from Ensembl Compara (Herrero et al., 2016), bringing the number of $S$. japonicus proteins with identified $S$. pombe orthologs to 4504. To find missing divergent orthologs, we identified proteins that are conserved between $S$. pombe and other species but did not have identified orthologs in S. japonicus, and devised gene-specific search strategies using a combination of FASTA (Pearson and Lipman, 1988), JackHMMER (Johnson et al., 2010), PSI-BLAST (Altschul et al., 1997), TBLASTN (Gertz et al., 2006), and synteny. This procedure also identified missing genes and gene structures that required revision. 
Human and budding yeast orthologs. In PomBase, orthologs 218 between $S$. pombe and Saccharomyces cerevisiae (budding 219 yeast) and between $S$. pombe and human have been manually220 curated over twenty years using multiple ortholog prediction 221 methods and tailored search strategies to provide complete and highly accurate coverage of known orthologs, including 22 many not identified by automated methods (Wood, 2005; $\mathrm{Hu}_{223}$ et al., 2011). We inferred human and budding yeast orthologs ${ }_{224}$ by transferring orthologs manually curated by PomBase for $S_{.225}$ pombe genes to the orthologous $S$. japonicus genes. These were ${ }_{226}$ supplemented with orthologs from Compara for proteins that ${ }_{227}$ have no $S$. pombe ortholog but are conserved in other species. 228

Families and domains

229 Protein domain information from InterPro version 85 (Blum $^{231}$ et al., 2021), together with coiled-coil and low-complexity ${ }^{232}$ regions from Pfam version 34.0 (El-Gebali et al., 2019), ${ }^{233}$ were imported using existing PomBase Chado loading code. ${ }^{234}$ Transmembrane domains were predicted using TMHMM $^{235}$ (Krogh et al., 2001).

\section{Gene Ontology} UniProt file produced by the Gen project at EBI (Huntley et al., 2015), and then filtering for NCBI taxon ID 4897 or 402676 . The loading script checks 243 the GOA UniProt file (located at ftp://ftp.ebi.ac.uk/pub/244 databases/GO/goa/UNIPROT/goa_uniprot_all.gaf.gz) for updates 245 upon each run. For the first JaponicusDB load, we used the GOA file from July 2021, which contains 36878 S. japonicus ${ }^{246}$ annotations.

The filtering protocol used by PomBase to retain only ${ }^{248}$ relevant and non-redundant automated annotation, in which ${ }^{249}$ GO annotations are filtered at two stages, was applied ${ }^{250}$ (Lock et al., 2018). Prior to loading into Chado, the GOA $^{251}$ annotations were filtered to exclude any derived from false ${ }^{252}$ positive or otherwise inapplicable InterPro2GO (Finn et al. ${ }^{253}$ 2017, https://github.com/geneontology/go-site/blob/master/ metadata/gorefs/goref-0000002.md) and keyword (https://254 github.com/geneontology/go-site/blob/master/metadata/gorefs/ goref-0000041.md, https://github.com/geneontology/go-site/ ${ }_{256}$ $\mathrm{blob} / \mathrm{master} / \mathrm{metadata} /$ gorefs/goref-0000043.md) mappings, or ${ }_{257}$ phylogeny-based transfer (Gaudet et al., 2011), and any ${ }_{258}^{257}$ GO terms that are flagged by GO as not usable for direct ${ }_{259}$ annotation, or as inapplicable due to taxon restrictions (Deegan ${ }_{260}$ née Clark et al., 2010; The Gene Ontology Consortium, 2021); ${ }_{261}$ after filtering, 24159 annotations were loaded.

To enhance the S. japonicus GO annotation set, S. pombe GO annotations were transferred to $S$. japonicus orthologs, and manual annotations from literature curated in Canto were ${ }^{263}$ added. The annotations loaded into Chado were then subjected264 to the second round of filtering, which removes redundant265 non-experimental GO annotation.

The JaponicusDB GO pipeline also supports configurable267 filtering that can be used in cases where paralogs have268 diverged (e.g. one or both of a paralogous pair has undergone269 neofunctionalisation or sub-functionalisation), making the270 S. pombe annotation inappropriate for S. japonicus; with 271 automated annotation transfer suppressed, the paralogs can 272 be manually annotated. Annotation from PomBase is also not273 transferred for any gene-term combination that contradicts a274 manually curated negated (NOT) annotation in JaponicusDB275 (e.g. Mid1 GO:1902408). Finally, for known proteins conserved276 in other species but absent from $S$. pombe, GO annotations were made manually by inference from experimentally characterised orthologs. As of September 2021, JaponicusDB has 29589 GO annotations.

Identification of missing genes and revision of gene structures Protein-coding genes. During manual ortholog assignment, we observed that a number of genes at syntenic locations did not display any clear sequence similarity. Inspecting the structures of these genes revealed errors, which were corrected. All gene structure editing was done using the Artemis genome annotation tool (Rutherford et al., 2000). Gene structures annotated in the published assembly without methionines were either trimmed to the appropriate methionine, or revised to include a missing N-terminal exon. Gene structure errors reported in publications were also corrected (Makarova et al., 2016, 2020).

We created a list of $S$. pombe genes that are broadly conserved in single copy throughout eukaryotes (predominantly one-to-one to human) using the PomBase advanced search and imported the result list into JaponicusDB using the identifier mapping feature (Harris et al. in this issue). This query combination provided a list of conserved proteins present in $S$. pombe but absent from $S$. japonicus. Since we expected most of these genes to be present in S. japonicus, we used TBLASTN to perform directed searches against the $S$. japonicus genome, using the $S$. pombe proteins as input, coupled with manual inspection and gene structure curation in Artemis to identify highly spliced genes at syntenic locations.

Non-coding RNAs. Annotated features imported with the genome sequence included 347 tRNA genes and 17 rRNA genes, but no other non-coding RNAs. We used tRNAscan-SE (Chan and Lowe, 2019) to confirm the tRNA complement, identifying three additional tRNA genes (SJAG_08001-SJAG_08003), and as a source of codon information for the product descriptions. Other missing non-coding RNAs were imported from RNAcentral (The RNAcentral Consortium, 2019).

\section{Website code and documentation}

The JaponicusDB website uses the PomBase website code (Lock et al., 2018), configured for S. japonicus. In brief, the Chado database is processed nightly to build a Docker container which holds a complete instance of the website, including all documentation, HTML, code, and data.

All documentation for JaponicusDB is maintained in Markdown format in a GitHub repository and the source files are shared with PomBase where possible.

\section{Canto setup and literature import}

The Canto community curation tool was designed to support different species and datatypes via simple configuration adjustments (Rutherford et al., 2014). As deployed for $S$. japonicus, Canto currently supports annotating phenotypes using FYPO, alleles and genotypes, protein modifications, genetic and physical interactions, qualitative gene expression, and GO, including associated annotation extensions (Huntley et al., 2014; Lock et al., 2018) and metadata. A PubMed search using a set of $S$. japonicus-related keywords retrieved 179 publications, which were imported into Canto along with all citation details needed to populate Canto and the website. Publications containing gene-specific data were flagged using Canto's literature triage function, which allows users 
to classify papers by type (e.g., curatable, review, methods),337 and then assign them to authors for full curation. The triage 38 procedure also flagged 91 false positives, most of which wereз39 publications describing a "japonicus" species in a genus other340 than Schizosaccharomyces. We have refined the PubMed search341 keywords based on these results, so future Canto publication 342 updates will include fewer false positives.

\section{Results}

Adding value to imported data

Adding and revising gene structures

The original gene models for $S$. japonicus were built using ${ }^{348}$ aligned RNA-seq transcripts from S. pombe, S. cryophilus and ${ }_{350}^{349}$ $S$. octosporus orthologous groups compared to protein sequence (Rhind et al., 2011). Gene models with discrepancies were ${ }_{352}$ then manually reviewed to provide a set of high-quality gene ${ }_{353}$ predictions. However, continual revisions are required to make corrections and keep gene structures in line with published ${ }_{355}$ knowledge.

In this work, we used previously published information ${ }^{356}$ (Makarova et al., 2016) and detailed orthology inspection to ${ }_{358}^{357}$ revise 36 gene structures, illustrated in Figure 1. We identified ${ }_{359}$ 18 additional conserved genes (SJAG_07000-SJAG_07017) by ${ }_{360}$ searching for small, universally conserved proteins absent from ${ }_{361}$ the predicted gene set. In eight cases, we replaced erroneous ${ }_{362}$ gene predictions with genes correctly identified in an alternative ${ }_{363}$ reading frame. Newly identified genes, include Atp19, an F-type ATPase subunit for which no gene structure previously existed 364 (Figure 1A), and the recombination protein Rec7, for which the365 originally predicted structure was completely replaced by a new366 structure (Figure 1B). For SJAG_03830, the predicted structure367 was revised but not completely replaced (Figure 1C), allowing 368 the gene product to be identified as the ubiquinol-cytochrome-369 c reductase complex subunit Qcr10, previously thought absent370 from S. japonicus.

Using tRNAscan-SE (Chan and Lowe, 2019), we added372 three genes to the tRNA complement, and added product373 descriptions specifying codons to the 325 nuclear-encoded 374 tRNAs. Additional non-coding RNAs including snRNAs,375 snoRNAs, and telomerase RNA were imported from RNAcentrak76 (The RNAcentral Consortium, 2019).

\section{Identifying distant orthologs}

Accurate detection of orthologs, i.e. genes in different ${ }^{380}$ species related by vertical descent (Fitch, 1970), between ${ }^{381}$ two proteomes allows for comprehensive comparative study of ${ }^{382}$ the biological processes in two species. Orthologs provide $\mathrm{a}^{383}$ framework for reconstructing the evolutionary events that have ${ }^{384}$ given rise to observed biological differences. Thorough ortholog ${ }^{385}$ inventories require the targeted detection of distant orthologs ${ }^{386}$ and the identification of the orthologous relationship type $\mathrm{e}^{387}$ (i.e., one-to-one, one-to-many, or many-to-many). Ortholog ${ }^{388}$ inventories also facilitate the accurate identification of lineage ${ }^{389}$ specific gene losses, species-specific genes, and protein family ${ }^{390}$ expansions.

In this study, we identified 67 previously undetected distant ${ }^{392}$ orthologs between $S$. pombe and $S$. japonicus, 25 of which are ${ }^{393}$ also conserved in human (Supplementary Table S1). In two cases ortholog detection depended on the revision of existing 394 gene structures and in 18 cases, finding new genes. Candidates395 for a further 17 orthologs (including the kinetochore regulator396 Meikin, the kinetochore protein Mis19, and the spindles97 component Dms1) are recorded as high confidence based on a one-to-one relationship between $S$. pombe and other species and syntenic location between $S$. pombe and $S$. japonicus, although sequence similarity has not yet been detected. These regions are currently being reviewed for possible gene prediction errors. Finally, some additional ortholog calls from the automated pipelines were revised to include in-paralogs and remove outparalogs.

\section{Gene names and product descriptions}

Gene names. In consultation with S. japonicus researchers, we have assigned standard gene names in JaponicusDB, using unified nomenclature to make conserved loci readily recognisable and to avert potential naming conflicts with other species. Only 101 gene names were imported from UniProtKB for $S$. japonicus. Names for 3471 protein-coding genes were assigned by importing $S$. pombe names from PomBase for all clear one-to-one orthologs. Manual curation of 119 exact or near-exact duplicate proteins (ribosomal proteins, histones, translation elongation factors) enabled us to assign names for syntenic orthologs. Finally, 184 gene names were assigned manually to $S$. japonicus specific families, one-to-many, and many-to-one paralogs. Overall, JaponicusDB now provides standard names for 3875 protein-coding genes (of 4896 total). The remit of the fission yeast Gene Naming Committee (GNC; https://www.pombase.org/submit-data/gene-names) has expanded to cover $S$. japonicus as well as $S$. pombe gene names; the GNC will approve all new gene names in both species.

Gene product descriptions. Informative gene product descriptions allow users to browse proteomes effectively, scan input and output lists from experiments, and review genome contents. Gene product descriptions for 4251 protein-coding genes were automatically transferred from one-to-one orthologs curated by PomBase. A further 645 descriptions were manually curated based on one-to-many, many-to-one, or many-to-many orthologs or protein family descriptions. JaponicusDB now provides gene product labels that have been reviewed by fission yeast experts, and accurately reflect current knowledge for 4896 protein-coding genes.

Our analysis and curation pipeline has improved gene product descriptions dramatically compared to previously available data. UniProtKB provides descriptions for protein gene products, but over $99 \%$ of $S$. japonicus proteins remain in the unreviewed TrEMBL database and therefore have only automated descriptions. The quality of TrEMBL's inferred descriptions is generally high, but coverage is incomplete. Notably, we have provided informative product labels for 522 proteins described as "uncharacterized protein" in UniProtKB. Many of these are broadly conserved, well-studied proteins identified and annotated via our distant ortholog detection or manual curation pipelines. For example, we found five proteins involved in TOR signalling and three cytochrome oxidase assembly factors.

The JaponicusDB pipeline updates imported gene names and product descriptions upon each run. Simple configuration files maintained in GitHub repositories specify manually curated names and descriptions that take precedence over automated imports.

Extending GO annotation coverage and specificity To generate a Gene Ontology (GO; The Gene Ontology Consortium 2000, 2021) annotation dataset for S. japonicus, we imported computationally generated annotations from 
the GOA UniProt (Huntley et al., 2015), and added new460 annotations from the PomBase ortholog pipeline, manual461 inferences for genes without S. pombe orthologs, and462 experimental data from literature manually curated in Canto.463 We applied the filtering protocol used by PomBase (Lock464 et al., 2018) to retain only relevant and non-redundant 465 automated annotation. Annotations manually curated from the466 literature in Canto take precedence over any automatically467 transferred annotations (for example, as noted in Methods, manual negated annotations suppress import of contradictory 468 automated annotations). The JaponicusDB GO annotation procedures thus support robust transfer of large annotation ${ }_{470}$ sets, while also allowing the fine tuning of specific annotation to capture known biological differences (reviewed in Gu and ${ }^{471}$ Oliferenko 2015; Russell et al. 2017; Oliferenko 2018; also see ${ }_{473}^{472}$ references cited in Introduction). The resulting GO annotation ${ }_{474}^{473}$ dataset comprises 29589 annotations as of September 2021.

PomBase maintains subsets of GO, known as "GO slims", of selected biologically meaningful terms from each branch of GO - Molecular Function (MF), Biological Process (BP), and ${ }^{47}$ Cellular Component (CC) - that are used to summarise the functional capabilities of S. pombe (Lock et al., 2018; Wood et al., 2019). JaponicusDB uses the PomBase GO slims for ${ }^{48}$ the same purposes, illustrating the value added by our $\mathrm{GO}^{481}$ curation procedures. GO slim analysis shows that annotation ${ }^{482}$ breadth has significantly increased compared to the GO data ${ }_{484}^{433}$ originally available from GOA despite an overall decrease in annotation number (from 36878 to 29512). For each aspect ${ }_{486}^{45}$ of GO, more genes are annotated to terms in the GO $\operatorname{slim}^{480}$ (MF coverage increased from 3087 to 3652 , BP from $3238^{487}$ to 4187 , and CC from 2999 to 4578 genes), and the number of genes that either have no annotation, or are annotated ${ }^{489}$ but not covered by the GO slim, is correspondingly decreased ${ }^{490}$ (Figure 2). Notably, we have increased annotation coverage in several areas of active $S$. japonicus research. For example, the ${ }_{491}$ annotation increases for "membrane organization" and "lipid metabolism" in BP and "endoplasmic reticulum" (ER) and ${ }^{492}$ "mitochondrion" in CC are highly relevant to comparative ${ }^{493}$ studies of respiration and membrane and lipid biology. In MF, 494 there is a large increase in annotations to "hydrolase activity" ${ }^{495}$ including 21 gene products annotated to "lipid metabolism". ${ }^{496}$ Newly annotated examples in this set include the mitochondrial ${ }^{497}$ cardiolipin-specific phospholipase Cld1, the acyl-coenzyme A ${ }^{498}$ thioesterase The4, and SJAG_00199, a mitochondrial DDHD ${ }^{499}$ family phospholipase. The relatively new GO term "molecular ${ }^{500}$ adaptor activity", relevant to research in organelle organisation, ${ }^{501}$ also has increased annotations including many newly described ${ }^{502}$ protein- and organelle-to-membrane adaptors.

Annotation depth, defined as increased distance from the ${ }^{504}$ root node in an ontology graph, provides a measure of the ${ }^{505}$ biological specificity of the annotations. Our work has increased ${ }^{506}$ annotation depth in at least one GO aspect (Molecular ${ }^{507}$ Function, Biological Process or Cellular Component) for $4312^{508}$ proteins ( $>88 \%$ of the total), and in all 3 aspects for $1385^{509}$ $(>28 \%)$ (Figure 3$)$.

Using Canto's literature triage function, we have manually513 classified the 179 publications found in our initial PubMed514 query (see Methods). After discarding spurious matches, we515 identified 31 papers containing gene-specific data suitable for516 curation, as well as 39 papers in other categories. The latter517 reflect the status of $S$. japonicus as an emerging model, and518 include publications describing wild type features and cell composition, methods and reagents, or phylogenetic studies, as well as reviews. The 31 curatable papers were assigned to the authors (or to a Canto administrator) for curation, and 23 are now fully curated in JaponicusDB (see https://www. japonicusdb.org/reference_list/community). To date, manual curation has supplied 135 experimentally supported GO annotations and 168 phenotype annotations.

\section{Website}

The JaponicusDB website, modelled on PomBase, provides convenient access to all molecular and cell biological data curated for S. japonicus. Like PomBase, JaponicusDB includes pages for each gene, publication, and genotype annotated, as well as for ontology terms. JaponicusDB also uses the same simple, advanced, and peptide motif search tools as PomBase. To facilitate comparison between S. japonicus and S. pombe, the "Ortholog" section of each gene page includes reciprocal links between PomBase and JaponicusDB. On both sites, orthologs can also be retrieved via the advanced search or via an ID mapping tool. The JaponicusDB front page (Figure 4) presents news, database usage hints, publications recently curated by the community, and "Research Spotlight" panels, which feature graphical abstracts from recent papers (one of the most popular features of PomBase). An instance of the genome browser JBrowse (Buels et al., 2016) currently displays the $S$. japonicus genome and annotated features, and will host any available high-throughput sequence-based datasets. Online documentation describes all page contents, search capabilities, and how to contribute to JaponicusDB (also see Lock et al. 2018 and Harris et al. in this issue). JaponicusDB uses Google Analytics to monitor website usage.

\section{Conclusions}

For several decades, fission yeast research has been a mainstay of progress in cell and molecular biology, due to pioneering work on cell cycle regulation, as well as recent innovative experimentation in additional areas such as chromosome segregation, epigenetics and cytokinesis, using S. pombe. The growing amount and sophistication of research on S. pombe's "sister" species S. japonicus provides a prime opportunity to add evolutionary biology to the roster of topics that can be fruitfully investigated using fission yeast.

We have launched JaponicusDB to enable S. japonicus researchers - and the wider scientific community - to realise the full promise of experiments in an emerging model system. JaponicusDB gathers and interconnects diverse types of information from many sources to create a single resource that provides coherent, intuitive access to rich, interconnected data sets. As a fully functional MOD, JaponicusDB supports robust genome-wide data interrogation and mining at any level of detail, experimental planning, hypothesis generation, and interpretation of results, thereby meeting research needs not addressed by any other system.

\section{PomBase code re-use}

We have re-used the modular, configurable open-source code underlying PomBase, enabling us to deploy a curation database, a Canto instance, and a functioning, intuitive website for JaponicusDB with modest developer and curator input. (We estimate that essential development took the equivalent of approximately $1 \mathrm{FTE}$ for six weeks, and we 
devoted a similar amount of time to curation, i.e. 12578 FTE-weeks total.) For updates, the PomBase system relies579 primarily on data and configuration files stored (with version580 control) in GitHub repositories, making future maintenance581 straightforward. Because JaponicusDB uses PomBase code,582 both databases will simultaneously deploy any new features583 developed by the PomBase team.

\section{Community literature curation}

The Canto community literature curation system enables bench ${ }_{587}^{587}$ biologists to contribute directly to the knowledge integrated ${ }_{589}$ into JaponicusDB. Although Canto is also being used for ${ }_{590}$ phenotype curation in FlyBase (Larkin et al., 2021) and $_{591}$ pathogen-host interaction phenotypes in PHI-Base (Urban ${ }_{592}$ et al., 2020), JaponicusDB has embraced Canto for community ${ }_{593}$ curation at a uniquely early stage in its development. The ${ }_{594}$ curatable literature corpus is small at present, but over $_{595}$ two thirds of suitable papers have already been curated. ${ }_{596}$ $S$. japonicus researchers can use community curation to meet data dissemination objectives, helping ensure timely FAIR (Findable, Accessible, Interoperable and Reusable;597 Wilkinson et al. 2016) sharing of new information. $\mathrm{To}_{598}$ enhance engagement with JaponicusDB and interactions among ${ }_{599}$ community members, a mailing list has been set up (https: 600 //mailman.kcl.ac.uk/mailman/listinfo/japonicus-list).

\section{Community MOD management}

To ensure that JaponicusDB meets its users' needs in the ${ }^{604}$ future, we will invite experts in relevant areas, including $S .{ }^{605}$ japonicus biology and database curation and maintenance, ${ }^{606}$ to form a Scientific Advisory Board (SAB). The SAB will ${ }^{607}$ collaborate with PomBase staff to mobilise volunteers from ${ }^{608}$ the $S$. japonicus community to carry out routine updates ${ }^{609}$ and maintenance, and will guide future fission yeast MOD $^{610}$ developments that arise from $S$. japonicus research. PomBase ${ }^{611}$ staff will provide training and advice to community volunteers ${ }^{612}$ in configuring the website and JBrowse track metadata, Canto ${ }^{613}$ administration, editing gene structures, and other maintenance ${ }^{614}$ tasks. This group will also arrange to disseminate data regularly ${ }^{6}$ from JaponicusDB to external resources, e.g. GO annotations ${ }^{616}$ to the GO Consortium repository.

\section{Curation to support $S$. japonicus research}

Our review of the $S$. japonicus genome highlights the value that 621 manual curation adds to sequence feature annotation, ortholog 622 identification, and functional annotation, and therefore to623 all subsequent usage of these data in experimental and predictive studies. As a result, JaponicusDB can provide more comprehensive, experimentally informed complements ${ }_{624}$ of several types of genome-scale data than were previously available via data aggregators. We note that finding genes ${ }^{625}$ previously thought to be absent from $S$. japonicus has ${ }^{626}$ significant consequences for accurately representing molecular ${ }^{627}$ and cellular processes within the species as well as comparative ${ }^{628}$ studies of how these processes have evolved.

Gene structures for most genomes are derived by automated ${ }^{630}$ prediction pipelines, often incorporating information from $^{631}$ homology and synteny. Although multiple methods are ${ }^{632}$ frequently used, many errors will persist even for intensively ${ }^{633}$ curated model species in which all gene predictions were ${ }^{634}$ manually inspected prior to publication. For example, since publication of the $S$. pombe genome, PomBase has revised
$>200$ gene structures; similarly, WormBase has updated several thousand for C. elegans (pers. comm. Paul Davis, WormBase). Every genome-scale analysis depends upon comprehensive, manually refined gene structures (and often other sequence features) for accuracy, reproducibility, and relevance.

Our work also illustrates how biologists may benefit directly from manual ortholog curation. For example, we have identified S. japonicus orthologs of cytochrome c oxidase subunits Cox7 and Cox9, ATP synthase subunit Atp19, mitochondrial alphaketoglutarate dehydrogenase Ymr1, and ubiquinol-cytochromec reductase complex subunit Qcr10, all of which are important for fission yeast researchers studying the differences in central carbon metabolism between $S$. pombe and S. japonicus.

Future updates to ortholog predictions, gene structures, names, and product descriptions, are bound to reveal more examples where comprehensive, curated knowledge is indispensable for understanding biological processes, avoiding misinterpretation, and making reliable cross-species comparisons

\section{Rapid MOD deployment to support emerging model species}

The rapid establishment of JaponicusDB promises to have beneficial repercussions well beyond the fission yeast community. First, simply having a full S. japonicus MOD available to facilitate and support research makes reliable information available to underpin comparative studies and data integration, not only between $S$. japonicus and $S$. pombe, but throughout all eukaryotes.

Perhaps of even greater consequence, JaponicusDB represents a proof of concept, demonstrating that a small group of people can easily deploy PomBase code to produce a comparable system for any other species. We and others have noted (Oliver et al., 2016; Alliance of Genome Resources Consortium, 2019; Lipshitz, 2021) that complete genome sequences continue to accumulate, but annotation even of sequence features, let alone any other associated data, cannot keep pace. A growing number of increasingly diverse research communities require creative approaches to data infrastructure to take advantage of the exploratory opportunities that a genome sequence offers, and to integrate genome-scale molecular data and methods with small-scale results from new or extant published literature. Our experience with JaponicusDB exemplifies one way forward: the PomBase system can be redeployed by a small, motivated community to create a resource that can be maintained within the scope of time and skills available to researchers, using funding allocated to data dissemination.

\section{Data Availability}

As described in detail above, JaponicusDB uses the opensource PomBase code base available from the PomBase GitHub organisation (https://github.com/pombase). JaponicusDB configuration and data files are available from the JaponicusDB GitHub organisation (https://github.com/japonicusdb).

JaponicusDB data can be viewed directly at https:// www.japonicusdb.org, query results provide data download options, and data can be downloaded in bulk from the website (see https://github.com/japonicusdb.org/datasets and https: //www.https://github.com/japonicusdb.org/data). 


\section{Acknowledgements}

We are grateful to the Wellcome Trust for allowing S. O. to ${ }^{693}$ redirect funds from a Senior Investigator Award (see below) ${ }^{694}$ to establish JaponicusDB. We thank Nick Rhind and Li-Lin ${ }^{695}$ $\mathrm{Du}$ for advice and discussions about the $S$. japonicus genome ${ }^{696}$ sequence, Blake Sweeney of the RNAcentral Consortium ${ }^{697}$ for help with non-coding RNA gene annotation, and the 698 $S$. japonicus researchers who have contributed community ${ }^{699}$ curation (Sophie Martin, Makoto Kawamukai, Elisa Gomez-Gil, ${ }_{701}^{700}$ and Ying $\mathrm{Gu})$.

\section{Funding}

This research was funded in whole, or in part, by the Wellcome ${ }_{706}^{705}$ Trust [Grant number 103741/Z/14/Z to S. O.]. For the purpose of open access, the author has applied a CC BY public copyright ${ }_{708}$ licence to any Author Accepted Manuscript version arising from ${ }_{709}$ this submission.

S. O. also acknowledges funding from Wellcome Trust ${ }_{711}$ (Investigator Award in Science $[220790 / \mathrm{Z} / 20 / \mathrm{Z}]$ ) and $\mathrm{BBSRC}_{712}^{711}$ [BB/T000481/1]. The Francis Crick Institute receives its ${ }_{713}$ core funding from Cancer Research UK (FC001002), the UK ${ }_{714}$ Medical Research Council (FC001002), and the Wellcome Trust ${ }_{715}$ (FC001002). PomBase is supported by the Wellcome Trust ${ }_{716}$ [218236/Z/19/Z to Juan Mata].

\section{Conflicts of interest}

None declared.

\section{References} Alliance of Genome Resources Consortium. The Alliance of 725 Genome Resources: Building a modern data ecosystem for726 model organism databases. Genetics, 213(4):1189-1196,727 2019.

728

S. F. Altschul, T. L. Madden, A. A. Schäffer, J. Zhang, 729 Z. Zhang, W. Miller, and D. J. Lipman. Gapped BLAST730 and PSI-BLAST: a new generation of protein database search731 programs. Nucleic Acids Res, 25(17):3389-3402, 1997. K. Aoki, H. Hayashi, K. Furuya, M. Sato, T. Takagi, M. Osumi,733 A. Kimura, and H. Niki. Breakage of the nuclear envelope 734 by an extending mitotic nucleus occurs during anaphase in735 Schizosaccharomyces japonicus. Genes Cells, 16(9):911-926,736 2011.

E. Y. Basenko, J. A. Pulman, A. Shanmugasundram, O. S.738 Harb, K. Crouch, D. Starns, S. Warrenfeltz, C. Aurrecoechea,739 C. J. Stoeckert, J. C. Kissinger, D. S. Roos, and C. Hertz-740 Fowler. FungiDB: An integrated bioinformatic resource for741 fungi and oomycetes. J Fungi (Basel), 4(1), 2018. M. Blum, H.-Y. Chang, S. Chuguransky, T. Grego,743 S. Kandasaamy, A. Mitchell, G. Nuka, T. Paysan-Lafosse,744 M. Qureshi, S. Raj, L. Richardson, G. A. Salazar,745 L. Williams, P. Bork, A. Bridge, J. Gough, D. H. Haft,746 I. Letunic, A. Marchler-Bauer, H. Mi, D. A. Natale, M. Necci,747 C. A. Orengo, A. P. Pandurangan, C. Rivoire, C. J. A. Sigrist, 748 I. Sillitoe, N. Thanki, P. D. Thomas, S. C. E. Tosatto, C. H.749 $\mathrm{Wu}$, A. Bateman, and R. D. Finn. The InterPro protein 750 families and domains database: 20 years on. Nucleic Acids751 Res, 49(D1):D344-D354, 2021.

R. Buels, E. Yao, C. M. Diesh, R. D. Hayes, M. Munoz-Torres,753 G. Helt, D. M. Goodstein, C. G. Elsik, S. E. Lewis, L. Stein,754 and I. H. Holmes. JBrowse: a dynamic web platform for genome visualization and analysis. Genome Biol, 17:66, 2016.

C. J. E. A. Bulder. On respiratory deficiency in yeasts. $\mathrm{PhD}$ thesis, Technological University, Delft, Delft, The Netherlands, 1963.

C. E. Bullerwell, J. Leigh, L. Forget, and B. F. Lang. A comparison of three fission yeast mitochondrial genomes. Nucleic Acids Res, 31(2):759-768, 2003.

P. P. Chan and T. M. Lowe. tRNAscan-SE: Searching for tRNA genes in genomic sequences. Methods Mol Biol, 1962:1-14, 2019.

J. I. Deegan née Clark, E. C. Dimmer, and C. J. Mungall. Formalization of taxon-based constraints to detect inconsistencies in annotation and ontology development. BMC Bioinformatics, 11:530, 2010.

K. Eilbeck, S. E. Lewis, C. J. Mungall, M. Yandell, L. Stein, R. Durbin, and M. Ashburner. The Sequence Ontology: a tool for the unification of genome annotations. Genome Biol, 6 (5):R44, 2005

S. El-Gebali, J. Mistry, A. Bateman, S. R. Eddy, A. Luciani, S. C. Potter, M. Qureshi, L. J. Richardson, G. A. Salazar, A. Smart, E. L. L. Sonnhammer, L. Hirsh, L. Paladin, D. Piovesan, S. C. E. Tosatto, and R. D. Finn. The Pfam protein families database in 2019. Nucleic Acids Res, 47 (D1):D427-D432, 2019.

R. D. Finn, T. K. Attwood, P. C. Babbitt, A. Bateman, P. Bork, A. J. Bridge, H.-Y. Chang, Z. Dosztányi, S. El-Gebali, M. Fraser, J. Gough, D. Haft, G. L. Holliday, H. Huang, X. Huang, I. Letunic, R. Lopez, S. Lu, A. Marchler-Bauer, H. Mi, J. Mistry, D. A. Natale, M. Necci, G. Nuka, C. A. Orengo, Y. Park, S. Pesseat, D. Piovesan, S. C. Potter, N. D. Rawlings, N. Redaschi, L. Richardson, C. Rivoire, A. Sangrador-Vegas, C. Sigrist, I. Sillitoe, B. Smithers, S. Squizzato, G. Sutton, N. Thanki, P. D. Thomas, S. C. E. Tosatto, C. H. Wu, I. Xenarios, L.-S. Yeh, S.-Y. Young, and A. L. Mitchell. InterPro in 2017-beyond protein family and domain annotations. Nucleic Acids Res, 45(D1):D190-D199, 2017.

W. M. Fitch. Distinguishing homologous from analogous proteins. Syst Zool, 19(2):99-9113, 1970.

P. Gaudet, M. S. Livstone, S. E. Lewis, and P. D. Thomas. Phylogenetic-based propagation of functional annotations within the Gene Ontology consortium. Brief Bioinform, 12 (5):449-462, 2011.

E. M. Gertz, Y.-K. Yu, R. Agarwala, A. A. Schäffer, and S. F. Altschul. Composition-based statistics and translated nucleotide searches: improving the TBLASTN module of BLAST. BMC Biol, 4:41, 2006.

E. Gómez-Gil, A. Franco, M. Madrid, B. Vázquez-Marín, M. Gacto, J. Fernández-Breis, J. Vicente-Soler, T. Soto, and J. Cansado. Quorum sensing and stress-activated mapk signaling repress yeast to hypha transition in the fission yeast Schizosaccharomyces japonicus. PLoS Genet, 15(5): e1008192, 2019.

Y. Gu and S. Oliferenko. Comparative biology of cell division in the fission yeast clade. Curr Opin Microbiol, 28:18-25, 2015.

Y. Gu and S. Oliferenko. Cellular geometry scaling ensures robust division site positioning. Nat Commun, 10(1):268, 2019.

Y. Gu, C. Yam, and S. Oliferenko. Rewiring of cellular division site selection in evolution of fission yeasts. Curr Biol, 25(9): 1187-1194, 2015. 
M. A. Harris, A. Lock, J. Bähler, S. G. Oliver, and V. Wood.819 FYPO: the fission yeast phenotype ontology. Bioinformatics, 820 29(13):1671-1678, 2013.

P. W. Harrison, A. Ahamed, R. Aslam, B. T. F. Alako,822 J. Burgin, N. Buso, M. Courtot, J. Fan, D. Gupta, 823 M. Haseeb, S. Holt, T. Ibrahim, E. Ivanov, S. Jayathilaka, 824 V. B. Kadhirvelu, M. Kumar, R. Lopez, S. Kay, R. Leinonen, 825 X. Liu, C. O'Cathail, A. Pakseresht, Y. Park, S. Pesant,826 N. Rahman, J. Rajan, A. Sokolov, S. Vijayaraja, Z. Waheed,827 A. Zyoud, T. Burdett, and G. Cochrane. The Europeans28 Nucleotide Archive in 2020. Nucleic Acids Res, 49(D1):829 D82-D85, 2021

J. Hastings, G. Owen, A. Dekker, M. Ennis, N. Kale,831 V. Muthukrishnan, S. Turner, N. Swainston, P. Mendes, and832 C. Steinbeck. ChEBI in 2016: Improved services and an833 expanding collection of metabolites. Nucleic Acids Res, 44834 (D1):D1214-1219, 2016.

J. Herrero, M. Muffato, K. Beal, S. Fitzgerald, L. Gordon,836 M. Pignatelli, A. J. Vilella, S. M. J. Searle, R. Amode, 837 S. Brent, W. Spooner, E. Kulesha, A. Yates, and838 P. Flicek. Ensembl comparative genomics resources.839 Database (Oxford), 2016, 2016.

K. L. Howe, B. Contreras-Moreira, N. D. Silva, G. Maslen, 841 W. Akanni, J. Allen, J. Alvarez-Jarreta, M. Barba,842 D. M. Bolser, L. Cambell, M. Carbajo, M. Chakiachvili,843 M. Christensen, C. Cummins, A. Cuzick, P. Davis, S. Fexova, 844 A. Gall, N. George, L. Gil, P. Gupta, K. E. Hammond- 845 Kosack, E. Haskell, S. E. Hunt, P. Jaiswal, S. H. Janacek,846 P. J. Kersey, N. Langridge, U. Maheswari, T. Maurel, M. D.847 McDowall, B. Moore, M. Muffato, G. Naamati, S. Naithani,848 A. Olson, I. Papatheodorou, M. Patricio, M. Paulini,849 H. Pedro, E. Perry, J. Preece, M. Rosello, M. Russell,850 V. Sitnik, D. M. Staines, J. Stein, M. K. Tello-Ruiz, S. J.851 Trevanion, M. Urban, S. Wei, D. Ware, G. Williams, A. D.852 Yates, and P. Flicek. Ensembl Genomes 2020-enabling non-853 vertebrate genomic research. Nucleic Acids Res, 48(D1):854 D689-D695, 2020.

Y. Hu, I. Flockhart, A. Vinayagam, C. Bergwitz, B. Berger, 856 N. Perrimon, and S. E. Mohr. An integrative approach to857 ortholog prediction for disease-focused and other functionals58 studies. BMC Bioinformatics, 12:357, 2011.

R. P. Huntley, M. A. Harris, Y. Alam-Faruque, J. A. Blake, 860 S. Carbon, H. Dietze, E. C. Dimmer, R. E. Foulger, D. P.861 Hill, V. K. Khodiyar, A. Lock, J. Lomax, R. C. Lovering,862 P. Mutowo-Meullenet, T. Sawford, K. V. Auken, V. Wood,863 and C. J. Mungall. A method for increasing expressivity of 864 Gene Ontology annotations using a compositional approach.865 BMC Bioinformatics, 15:155, 2014.

R. P. Huntley, T. Sawford, P. Mutowo-Meullenet,867 A. Shypitsyna, C. Bonilla, M. J. Martin, and C. O'Donovan.868 The GOA database: Gene Ontology annotation updates for 869 2015. Nucleic Acids Res, 43(Database issue):D1057-1063,870 2015.

L. S. Johnson, S. R. Eddy, and E. Portugaly. Hidden Markov872 model speed heuristic and iterative HMM search procedure.873 BMC Bioinformatics, 11:431, 2010.

T. Kaino, K. Tonoko, S. Mochizuki, Y. Takashima, and875 M. Kawamukai. Schizosaccharomyces japonicus has low876 levels of CoQ10 synthesis, respiration deficiency, and efficient877 ethanol production. Biosci Biotechnol Biochem, 82(6):878 1031-1042, 2018.

879

C. Kinnaer, O. Dudin, and S. G. Martin. Yeast-to-hyphas80 transition of Schizosaccharomyces japonicus in response to881 environmental stimuli. Mol Biol Cell, 30(8):975-991, 2019. 882
A. Krogh, B. Larsson, G. von Heijne, and E. L. Sonnhammer. Predicting transmembrane protein topology with a hidden Markov model: application to complete genomes. J Mol Biol, 305(3):567-580, 2001.

A. Larkin, S. J. Marygold, G. Antonazzo, H. Attrill, G. dos Santos, P. V. Garapati, J. L. Goodman, L. S. Gramates, G. Millburn, V. B. Strelets, C. J. Tabone, J. Thurmond, and the FlyBase Consortium. FlyBase: updates to the Drosophila melanogaster knowledge base. Nucleic Acids Res, 49(Database issue):D899-D907, 2021.

H. D. Lipshitz. The descent of databases. Genetics, 217(3), 2021.

A. Lock, K. Rutherford, M. A. Harris, J. Hayles, S. G. Oliver, J. Bähler, and V. Wood. PomBase 2018: user-driven reimplementation of the fission yeast database provides rapid and intuitive access to diverse, interconnected information. Nucleic Acids Research, 47(D1):D821-D827, 2018. doi: 10.1093/nar/gky961. URL http://dx.doi.org/10.1093/nar/ gky961.

A. Lock, M. A. Harris, K. Rutherford, J. Hayles, and V. Wood. Community curation in PomBase: enabling fission yeast experts to provide detailed, standardized, sharable annotation from research publications. Database (Oxford), 2020:baaa028, 2020. ISSN 1758-0463. doi: 10.1093/database/ baaa028. URL https://doi.org/10.1093/database/baaa028.

M. Makarova, Y. Gu, J.-S. Chen, J. R. Beckley, K. L. Gould, and S. Oliferenko. Temporal regulation of lipin activity diverged to account for differences in mitotic programs. Curr Biol, 26(2):237-243, 2016.

M. Makarova, M. Peter, G. Balogh, A. Glatz, J. I. MacRae, N. L. Mora, P. Booth, E. Makeyev, L. Vigh, and S. Oliferenko. Delineating the rules for structural adaptation of membrane-associated proteins to evolutionary changes in membrane lipidome. Curr Biol, 30(3):367-380.e8, 2020.

L. Montecchi-Palazzi, R. Beavis, P.-A. Binz, R. J. Chalkley, J. Cottrell, D. Creasy, J. Shofstahl, S. L. Seymour, and J. S. Garavelli. The PSI-MOD community standard for representation of protein modification data. Nat Biotechnol, 26(8):864-866, 2008.

C. J. Mungall, D. B. Emmert, and FlyBase Consortium. A Chado case study: an ontology-based modular schema for representing genome-associated biological information. Bioinformatics, 23(13):i337-346, 2007.

S. Okamoto, K. Furuya, S. Nozaki, K. Aoki, and H. Niki. Synchronous activation of cell division by light or temperature stimuli in the dimorphic yeast Schizosaccharomyces japonicus. Eukaryot Cell, 12(9):12351243, 2013.

S. Oliferenko. Understanding eukaryotic chromosome segregation from a comparative biology perspective. $J$ Cell Sci, 131(14), 2018.

S. G. Oliver, A. Lock, M. A. Harris, P. Nurse, and V. Wood. Model organism databases: essential resources that need the support of both funders and users. BMC Biol, 14:49, 2016.

W. R. Pearson and D. J. Lipman. Improved tools for biological sequence comparison. Proc Natl Acad Sci U S A, 85(8): 2444-2448, 1988.

G. H. Pieper, S. Sprenger, D. Teis, and S. Oliferenko. ESCRT-III/Vps4 controls heterochromatin-nuclear envelope attachments. Dev Cell, 53(1):27-41.e6, 2020.

N. Rhind, Z. Chen, M. Yassour, D. A. Thompson, B. J. Haas, N. Habib, I. Wapinski, S. Roy, M. F. Lin, D. I. Heiman, S. K. Young, K. Furuya, Y. Guo, A. Pidoux, H. M. Chen, B. Robbertse, J. M. Goldberg, K. Aoki, E. H. 
Bayne, A. M. Berlin, C. A. Desjardins, E. Dobbs, L. Dukaj,947 L. Fan, M. G. FitzGerald, C. French, S. Gujja, K. Hansen,948 D. Keifenheim, J. Z. Levin, R. A. Mosher, C. A. Müller, 949 J. Pfiffner, M. Priest, C. Russ, A. Smialowska, P. Swoboda,950 S. M. Sykes, M. Vaughn, S. Vengrova, R. Yoder, Q. Zeng, R. Allshire, D. Baulcombe, B. W. Birren, W. Brown, K. Ekwall, M. Kellis, J. Leatherwood, H. Levin, H. Margalit, R. Martienssen, C. A. Nieduszynski, J. W. Spatafora, N. Friedman, J. Z. Dalgaard, P. Baumann, H. Niki, A. Regev, and C. Nusbaum. Comparative functional genomics of the fission yeasts. Science, 332(6032):930-936, 2011.

J. J. Russell, J. A. Theriot, P. Sood, W. F. Marshall, L. F. Landweber, L. Fritz-Laylin, J. K. Polka, S. Oliferenko, T. Gerbich, A. Gladfelter, J. Umen, M. Bezanilla, M. A. Lancaster, S. He, M. C. Gibson, B. Goldstein, E. M. Tanaka, C.-K. Hu, and A. Brunet. Non-model model organisms. BMC Biol, 15(1):55, 2017.

K. Rutherford, J. Parkhill, J. Crook, T. Horsnell, P. Rice, M. A. Rajandream, and B. Barrell. Artemis: sequence visualization and annotation. Bioinformatics, 16(10):944-945, 2000.

K. M. Rutherford, M. A. Harris, A. Lock, S. G. Oliver, and V. Wood. Canto: an online tool for community literature curation. Bioinformatics, 30(12):1791-1792, 2014

The Gene Ontology Consortium. Gene Ontology: tool for the unification of biology. Nat Genet, 25(1):25-29, 2000.

The Gene Ontology Consortium. The Gene Ontology resource: enriching a GOld mine. Nucleic Acids Res, 49(D1):D325D334, 2021.

The RNAcentral Consortium. RNAcentral: a hub of information for non-coding RNA sequences. Nucleic Acids Res, 47(D1):D221-D229, 2019.

The UniProt Consortium. UniProt: the universal protein knowledgebase in 2021. Nucleic Acids Research, 49(D1): D480-D489, 11 2020. ISSN 0305-1048. doi: 10.1093/nar/ gkaa1100. URL https://doi.org/10.1093/nar/gkaa1100.

M. Urban, A. Cuzick, J. Seager, V. Wood, K. Rutherford, S. Y. Venkatesh, N. D. Silva, M. C. Martinez, H. Pedro, A. D. Yates, K. Hassani-Pak, and K. E. Hammond-Kosack. PHIbase: the pathogen-host interactions database. Nucleic Acids Res, 48(D1):D613-D620, 2020.

M. D. Wilkinson, M. Dumontier, I. J. Aalbersberg, G. Appleton, M. Axton, A. Baak, N. Blomberg, J.-W. Boiten, L. B. da Silva Santos, P. E. Bourne, J. Bouwman, A. J. Brookes, M. Clark, T. Crosas, O. Dillo, I. Dumon, S. Edmunds, C. R. Evelo, T. Finkers, A. Gonzalez-Beltran, A. J. G. Gray, P. Groth, C. Goble, J. S. Grethe, J. Heringa, P. A. C. 't Hoen, T. Hooft, R. Kuhn, R. Kok, J. Kok, S. J. Lusher, M. E. Martone, A. Mons, A. L. Packer, B. Persson, M. Rocca-Serra, P. Roos, R. van Schaik, S.-A. Sansone, E. Schultes, T. Sengstag, T. Slater, G. Strawn, M. A. Swertz, M. Thompson, J. van der Lei, E. van Mulligen, J. Velterop, A. Waagmeester, P. Wittenburg, K. Wolstencroft, J. Zhao, and B. Mons. The FAIR Guiding Principles for scientific data management and stewardship. Sci Data, 3:160018, 2016.

V. Wood. Schizosaccharomyces pombe comparative genomics; from sequence to systems, pages 233-285. Springer, Berlin, Heidelberg, 2005. ISBN 978-3-540-31480-6.

V. Wood, A. Lock, M. A. Harris, K. Rutherford, J. Bähler, and S. G. Oliver. Hidden in plain sight: what remains to be discovered in the eukaryotic proteome? Open Biol, 9(2): 180241, 2019.

C. Yam, Y. He, D. Zhang, K.-H. Chiam, and S. Oliferenko. Divergent strategies for controlling the nuclear membrane satisfy geometric constraints during nuclear division. Curr
Biol, 21(15):1314-1319, 2011.

C. Yam, Y. Gu, and S. Oliferenko. Partitioning and remodeling of the Schizosaccharomyces japonicus mitotic nucleus require chromosome tethers. Curr Biol, 23(22):2303-2310, 2013. 


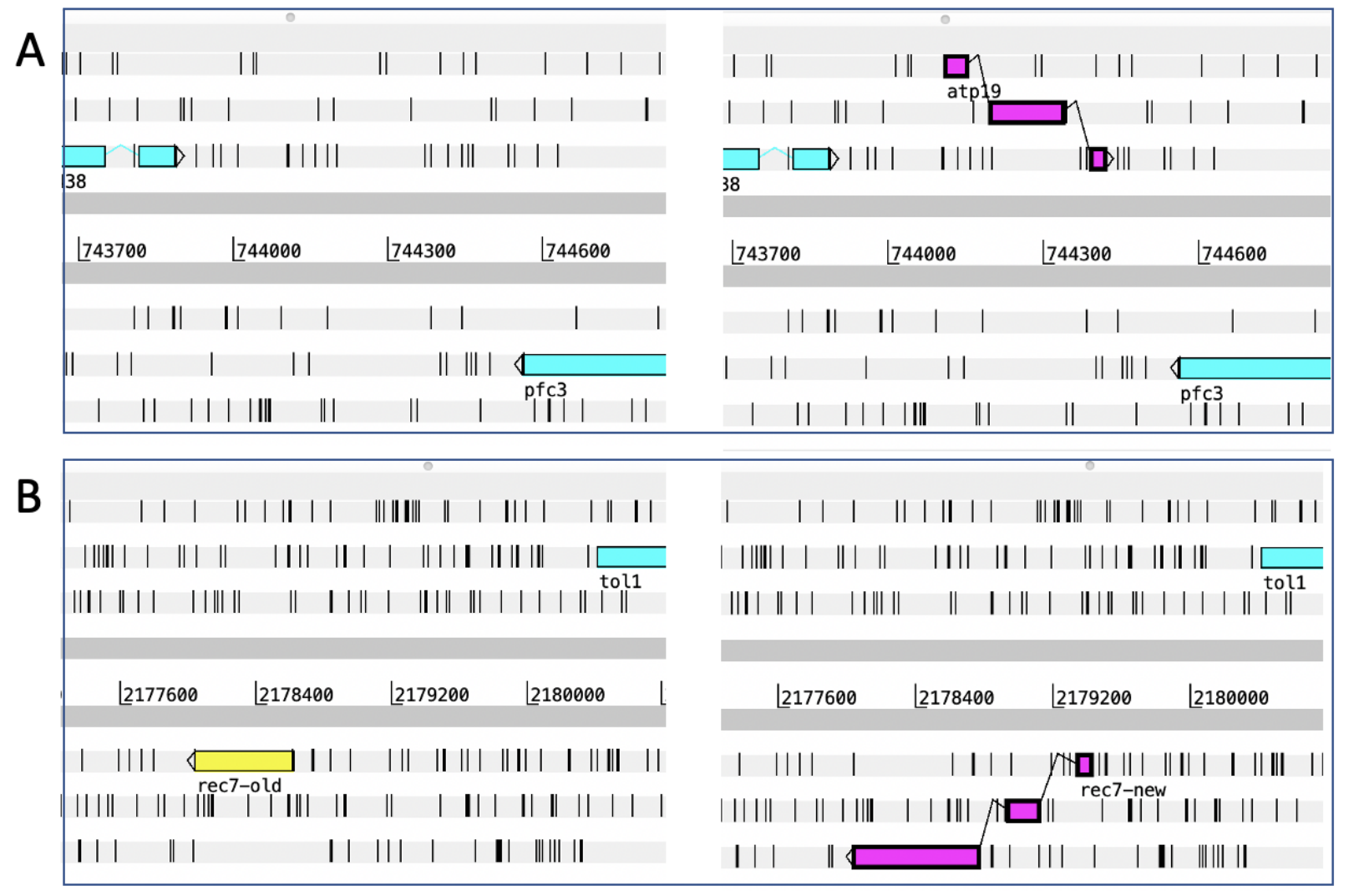

\begin{tabular}{|c|c|}
\hline 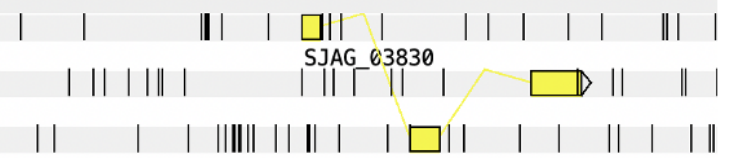 & 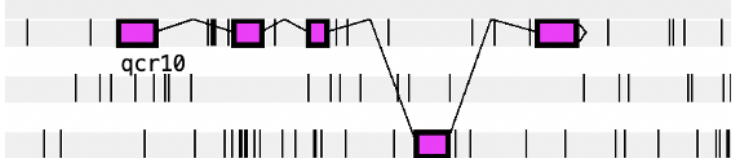 \\
\hline 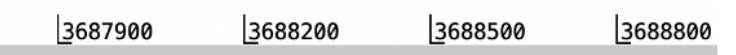 & 3688800 \\
\hline 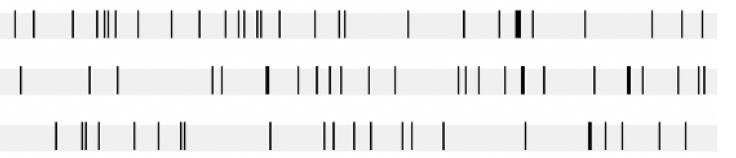 & 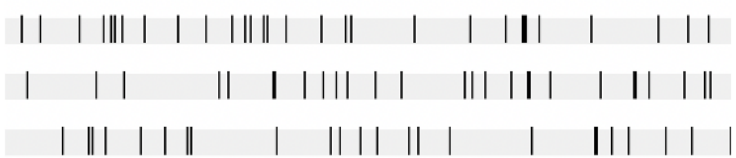 \\
\hline
\end{tabular}

Fig. 1. Examples of updated S. japonicus gene structures, illustrated using screenshots from the Artemis genome annotation tool (Rutherford et al., 2000). For each gene, the original genomic annotation is shown on the left and the revised annotation on the right. A) atp19, a newly identified gene; B) rec7, for which a new structure completely replaces the original structure; C) qcr10, for which the original structure was revised but not completely replaced. 

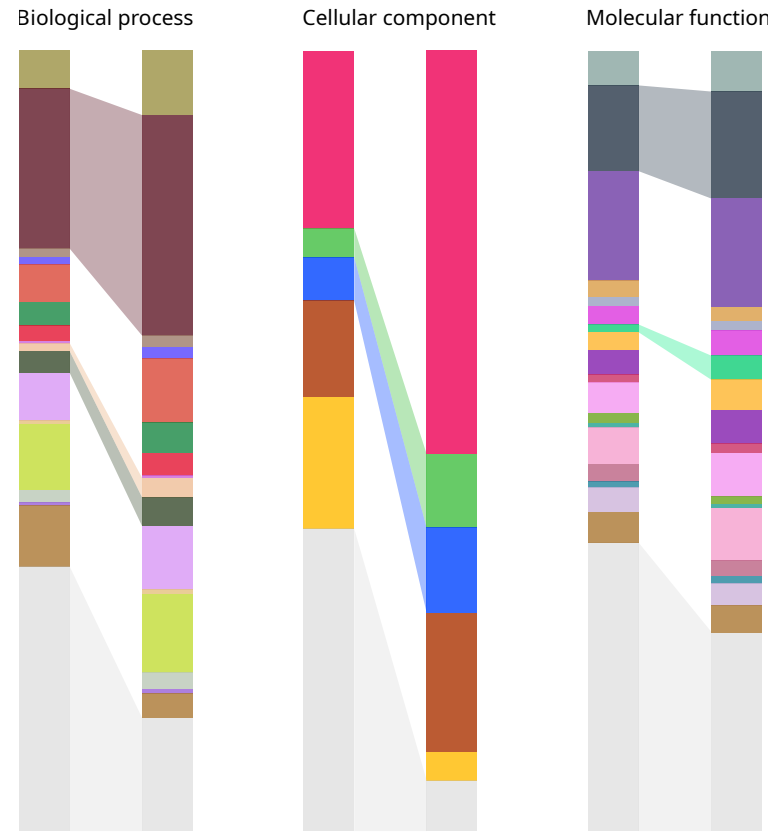

Process

signaling

gene expression

chromatin remod

protein folding

cellular component biogenesis

DNA metabolic process

cell cycle

cytoskeleton organization

membrane organizatio

lipid metabolic process

small molecule metabolic process

generation of precursor metabolites and energy

transport

cellular catabolic process

detoxification

other

other
unknown

Component

nucleus

endoplasmic reticulum

mitochondrion

cytoplasm

other

unknown

Fig. 2. Effect of curation on S. japonicus GO annotation coverage. Comparison of the distribution of annotations for all S. japonicus protein-coding genes (4896 total) to selected high-level terms in each aspect of GO between the UniProt GOA annotation file ("before"; left-hand columns) and the JaponicusDB GO annotation set ("after"; right-hand columns). The number of annotated proteins has increased for most Molecular Function (MF), Biological Process (BP), and Cellular Component (CC), with associated decreases in the proportions of proteins annotated as "unknown". Highlighted blocks represent GO terms relevant to processes that are intensively studied in S. japonicus: "gene expression", "membrane organization" and "lipid metabolism" in BP; "endoplasmic reticulum" and "mitochondrion" in CC; and "hydrolase activity" and "molecular adaptor activity" in MF. Images were generated using QuiLT (Harris et al., in this issue), in which only one term per gene can be included for display. If a gene is annotated to more than one GO term, one is selected according to a set order of precedence. Here, terms are arranged by order of precedence in the charts and key for each GO aspect. 


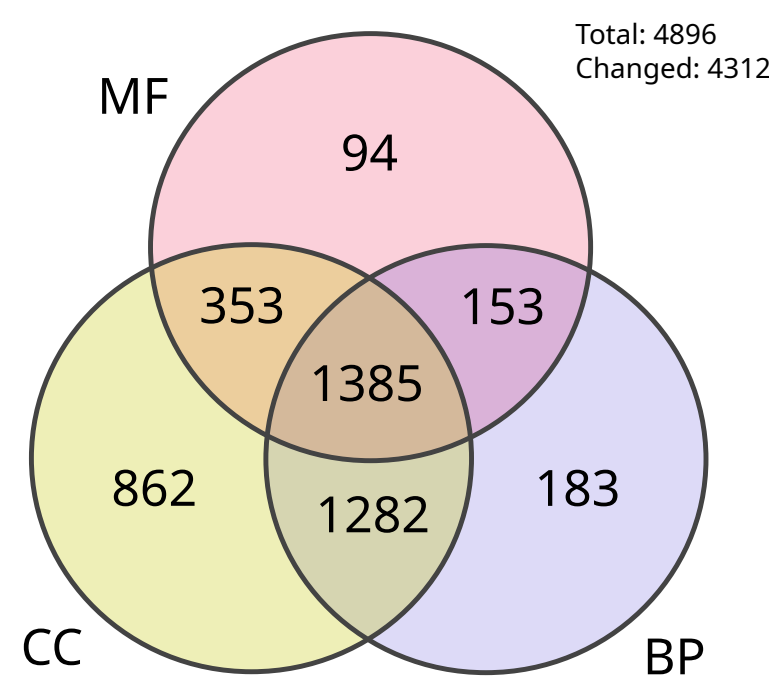

Fig. 3. S. japonicus proteins with increased GO annotation specificity. GO annotation specificity, defined as distance from the ontology root, was measured for each aspect of GO and for each of the 4896 proteins in S. japonicus. The Venn diagram shows the number of proteins that have more specific annotations in the JaponicusDB GO annotation set than in the UniProt GOA annotation file for one or more GO aspect(s) (MF, Molecular Function; BP, Biological Process; CC, Cellular Component). 
bioRxiv preprint doi: https://doi.org/10.1101/2021.09.23.461587; this version posted September 24, 2021. The copyright holder for this preprint (which was not certified by peer review) is the author/funder, who has granted bioRxiv a license to display the preprint in perpetuity. It is made available under aCC-BY 4.0 International license.

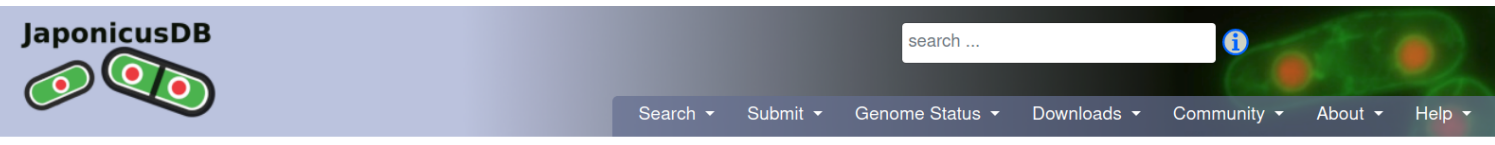

SQ $>0$ Contact curators...

\title{
Welcome to JaponicusDB
}

\begin{abstract}
This is the Schizosaccharomyces japonicus genome database Advanced search $\bullet$ Genome browser
Last updated: 2021-09-16

29825 annotations -23 curated publications

GO Overviews: Function • Process $\bullet$ Component

Example pages: Gene $\bullet$ Term $\bullet$ Publication
\end{abstract}

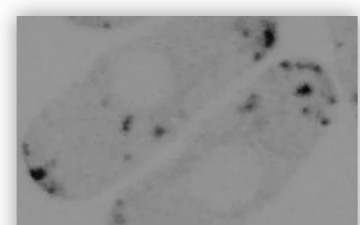

Research spotlight: Kinnaer et al. 2021

Yeast-to-hypha transition of

Schizosaccharomyces japonicus in

response to environmental stimuli.

Originally published in Mol Biol Cell.

Publication record in JaponicusDB ...

Archive

\section{Recent news}

So Official JaponicusDB release

2021-09-01

We are delighted to announce the official release of JaponicusDB a new, curated model activated MAPK signaling.

Quorum sensing and stress-activated MAPK signaling repress yeast

Anillin-related protein Mid1 regulates timely formation of the ...

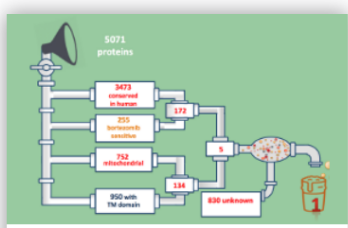

Explore JaponicusDB: Data mining Use the Advanced Search to construct complex queries (GO, phenotypes, taxonomic distribution, domain, chromosomal location).

Negative control of cytokinesis by stress-

\section{All community curation}

Tweets by @japonicusdb

国 JaponicusDB Retweeted

80 Gene Ontology

We'd like to officially welcome the

Fig. 4. The JaponicusDB front page. 